\title{
Genetic heterogeneity
}

National Cancer Institute ( $\mathrm{NCl})$

\section{Source}

National Cancer Institute (NCI). Genetic Heterogeneity.

The production of the same or similar phenotypes (observed biochemical, physiological, and morphological characteristics of a person determined by his/her genotype) by different genetic mechanisms. There are two types: (1) allelic heterogeneity - when different alleles at a locus can produce variable expression of a condition; and (2) locus heterogeneity - the term used to describe disease in which mutations at different loci can produce the same disease phenotype. 